\title{
Implementation and Evaluation of Manoeuvrable Blood Collection Nurse Posts
}

\author{
Haihua Yang, Guohua Huang* \\ Central Injection Room, The First Affiliated Hospital of Jinan University, Guangzhou, China \\ Email address: \\ yanghh200911@163.com (Haihua Yang),jndxhgh@163.com (Guohua Huang) \\ ${ }^{*}$ Corresponding author
}

To cite this article:

Haihua Yang, Guohua Huang. Implementation and Evaluation of Manoeuvrable Blood Collection Nurse Posts. European Journal of Clinical and Biomedical Sciences. Vol. 6, No. 1, 2020, pp. 14-17. doi: 10.11648/j.ejcbs.20200601.14

Received: January 14, 2020; Accepted: January 27, 2020; Published: February 12, 2020

\begin{abstract}
To study the practical effect of nursing fixed post and manoeuvrable post combined management mode in outpatient blood collection room. The construction process of joint management mode of fixed post and manoeuvrable post of nurses in blood collection room was introduced, including qualification examination, training, quality control, salary management and satisfaction evaluation of nurses in blood collection room, and the application effect compared before and after implementation. The waiting time for blood collection was reduced by 15.73 min during the peak period of blood collection in the outpatient blood collection room, and the satisfaction of patients with the service evaluation was significantly increased $(\mathrm{P}<0.01)$. Labor cost was reduced by 1.37 fixed positions while nurses' working enthusiasm was enhanced. The combined management mode of nursing fixed post and manoeuvrable post can shorten the waiting time for blood collection in the outpatient blood collection room during peak hours, improve patient satisfaction and save an power.
\end{abstract}

Keywords: Outpatient Blood Collection Room, Fixed Post, Manoeuvrable Post, Joint Management Mode, Effect Evaluation

\section{Introduction}

Manoeuvrable nurses are high-quality young nurses who are set up in the hospital nursing team under the unified deployment and management of the Nursing Department and have a certain degree of flexibility and emergency response [1]. The outpatient blood collection room serves as the hospital's service window. The quality of its nursing service and the level of business can directly affect the overall image of the hospital. The outpatient blood collection room is responsible for the collection of blood samples from outpatients. Most blood samples require fasting blood collection [2], the majority of the blood collection patients focus on collection in the morning, resulting in concentrated blood collection time., causing patients waiting for long peak hours, and other periods free. Patients wait longer during peak hours and complain more, which can easily lead to doctor-patient conflicts. Therefore, the problem of congestion during peak hours and shortening the waiting time of patients have become the first task that need to be resolved in the blood collection room. At present, most of the outpatient blood collection rooms adopt a fixed position management mode that is the hospital sets fixed positions for blood collection nurses according to the workload of the blood collection room. If there are no special circumstances, the number of positions will not be changed. The outpatient blood collection room of our hospital has adopted the management mode of combined manoeuvrable blood collection nurse posts and fixed-position nurses since March 2019, that is, in order to ensure the safety of patients and the quality of nursing services in the case of fixed-position blood collection nurses' work [3] cannot be completed in a timely manner. The hospital recruits manoeuvrable blood collection nurse positions to increase the total number of blood collection positions. This method can solve the problem of congestion during peak hours, save hospital labor costs, shorten patient waiting times, and improve patient satisfaction. It is reported as follows.

\section{Materials and Methods}

\subsection{General Information}

The outpatient blood collection room of our hospital belongs to the central injection room. There are five working 
areas in the department. In addition to the blood collection room, there are also adult infusion rooms, children's infusion rooms, intramuscular injection rooms, and catheter clinics. There are 16 nurses in the department, working seven hours a day, five working days a week, five people on holiday every day. Four to five nurses in the blood collection room during the morning, three nurses on weekends and holidays, and two in the afternoon. For blood collection, other nurses are responsible for Class A and Class $\mathrm{P}$ of the adult infusion room, children's infusion room, and intramuscular injection room. The age of the nurses in the department is 24-54 years old, with a median age of $(35 \pm 3.5)$ years. Education: 13 bachelor's degrees, 1 master's degree; Title: 1 deputy chief nurse, 6 supervisors, 7 nurses.

\subsection{Background of the Blood Collection Room's Position as a Manoeuvrable Blood Collection Nurse}

Since the opening of the second-child policy in China, the amount of blood collected in our outpatient clinic has increased year by year. At present, the average number of blood collection patients is 450 to 560 per day on the working day. There should be seven nurses. Due to lack of human resources, the waiting time for patients taking blood samples during peak hours in the morning is usually 30 to 40 minutes. The workload of the nurses is high, and the work is often delayed in the morning. The number of patients is relatively small, the workload is not enough, wasting manpower, and increasing manpower costs in the afternoon. Therefore, in order to optimize the blood collection process for outpatients, reduce the waiting time of patients, reduce labor costs, and apply for recruitment of manoeuvrable blood collection nurses from the hospital, the working day will increase two nurses, one in the morning on weekends, and the overtime expenses of the manoeuvrable personnel are paid according to the overtime of 4 hours a day.

\subsection{Construction of Post Management Mode for Manoeuvrable Blood Collection Nurses}

(1) Determine the entry standards for manoeuvrable blood collection nurses: a, obtain a nurse practice certificate, register in accordance with the regulations and be valid; $b$, participate in the hospital for 2 years and above, master the nursing operation skills, have a certain communication and coordination ability and Emergency rescue ability; c, participated in pre-job training, familiar with the environment of the blood collection room, blood collection technical specifications, work procedures, inspection items, precautions etc. (2) Develop the duties of manoeuvrable blood collection nurses: a, the manoeuvrable blood collection nurses will arrive at the office 10 minutes earlier to prepare for blood collection and environment preparation; $b$, strictly implement the check system to check patient information, including test items, test tube types, and paste barcodes; c, strictly implement the disinfection and isolation system and the principles of aseptic technology operation; $d$, explain the precautions and time and place of taking the test report to the patient before and after blood collection [4]. (3) Qualification review of enrolled nurses: the Nursing Department issued a notice in the hospital to recruit manoeuvrable blood collection posts in outpatient blood collection rooms. Nurses volunteered to register.

\subsection{Organize Nurse Training and Assessment}

The head of the blood collection room formulates the post duties, rules and regulations, blood collection room work procedures, and precautions to be grasped by the manoeuvrable blood collection nurse, and allows registered manoeuvrable blood collection nurses to learn by flipping the teaching method of the classroom [5]. All manoeuvrable blood collection nurses have participated in the three basic and three rigorous theoretical and technical trainings organized by the Nursing Department and have met the standards. Those who meet the assessment criteria are finally included in the manoeuvrable blood collection nurses bank, and a WeChat group of manoeuvrable blood collection nurses is established. At present, there are 250 manoeuvrable blood collection nurses.

\subsection{Quality Monitoring}

Establish a quality control team, composed of the head of the blood collection room and the backbone of the department, to perform quality control on the work of the manoeuvrable blood collection nurses, identify problems and correct them in a timely manner. The head nurse and the manoeuvrable blood collection nurse established a WeChat communication group. Any questions or suggestions from the department staff or the manoeuvrable blood collection nurse can be promptly reflected. The Nursing Department reviews the implementation status of the manoeuvrable blood collection nurses and the attendance of the manoeuvrable blood collection nurses every month, in order to find problems and analyze them, and propose corrective measures [6].

\subsection{Remuneration Management}

The overtime pay of manoeuvrable blood collection nurses is calculated based on 4 hours every morning. The attendance of the blood collection room nurses is statistically confirmed for attendance, submitted to the Nursing Department and the Personnel Department for review, and then submitted to the Finance Department for review.

\subsection{Recruitment and Post Arrangement of Manoeuvrable Blood Collection Nurses}

The head of the blood collection room sends the recruitment information of the manoeuvrable blood collection nurses next week on WeChat group on Friday. The manoeuvrable blood collection nurses enroll according to their rest time and energy without affecting the work of the undergraduate office. Finally, the head of the blood collection room determines the final recruitment list. According to the daily workload of our department, the manoeuvrable blood collection nurse post will be added during the morning peak hours on working days, and the blood collection room scheduling will be redesigned. The work hours will be increased from 7:30 to 11:30, and from 
8:00 to 12:00 during the morning peak hours on working days. One manoeuvrable blood collection nurse; one manoeuvrable blood collection nurse will be added from 8:00 to 12:00 during the non-working day peak hours, and the rest of the windows will be borne by fixed blood nurses in the blood collection room. The blood collection room is divided into two categories: blood collection post and barcode printing post. In order to reduce the occurrence of nursing errors, the manoeuvrable blood collection nurse is mainly responsible for the blood collection post. The key links such as barcode printing and sticking the barcode to the test tube are under the responsibility of the department nurse.

\subsection{Investigation of Waiting Time for Patients' Blood Collection}

Selected data of December 12-16, 2018 before implementation and August 12-16, 2019 after implementation, daily from 8:00 to 9:00, 9:00 to $10: 00$, and 10:00 to $11: 00$ From 11:00 to $12: 00$, the waiting time for blood collection of 10 patients in each survey is as follows: an investigator randomly records the time to the blood collection room on the patient's doctor's order guide when the patient reaches the barcode printing window. The staff wrote the end time of blood collection on the doctor's order guidance form, and the investigator retrieved the doctor's order guidance form and entered the results into the Excel software to establish a database, and perform classification and summary statistical analysis.

\subsection{Observation Indicators}

Compared human resource allocation before and after the implementation of manoeuvrable blood collection nurses, patient waiting time for blood collection, patient satisfaction, and nurse evaluation of manoeuvrable blood collection nurse post management; design patient satisfaction questionnaires by members of the quality control team, conduct patient satisfaction surveys regularly, and statistical analysis was performed after Wilcoxon rank sum test was performed.

\section{Results}

\subsection{Human Resources Allocation}

According to the number of working hours of fixed nurses in the week, 35 hours after the implementation of the manoeuvrable blood collection nurse post, the number of working hours of the fixed nurses has been reduced by an average of 48 hours a week, and about $1.37(48 \div 7 \div 5)$ fixed nurses. The hospital has saved labor costs, effectively alleviated the shortage of nursing staff, and improved the use efficiency of nursing staff.

\subsection{Patient Waiting for Blood Collection Time}

According to the survey results, the average waiting time for blood collection in the morning before implementation was $(25.14 \pm 6.60)$ minutes, and the average waiting time for blood collection after implementation was $(9.41 \pm 5.86)$ minutes. Patients' waiting time after implementation was shortened by 15.73 minutes than before (Table 1).

Table 1. Comparison of the average waiting time of patients during various peak hours before and after the implementation of manoeuvrable blood collection nurse positions.

\begin{tabular}{llllll}
\hline Time & $\mathbf{8 : 0 0} \mathbf{9 : 0 0}$ & $\mathbf{9 : 0 0} \mathbf{1 0 : 0 0}$ & $\mathbf{1 0 : 0 0} \mathbf{1 1 : 0 0}$ & $\mathbf{1 1 : 0 0 \sim 1 2 : 0 0}$ \\
\hline Before & 35.56 & 29.83 & 13.56 & 11.87 & $\mathbf{8 : 0 0 ~ 1 2 : 0 0}$ \\
After & 13.14 & 8.92 & 6.17 & 5.57 & 25.14 \\
Shorten time & 22.42 & 20.91 & 7.39 & 9.41 & 6.30 \\
\hline
\end{tabular}

\subsection{Increased Patient Satisfaction}

Consult the reference [7], self-designed the patient satisfaction questionnaire, including the environment of the outpatient blood collection room, the nurse's service attitude, blood collection technology and overall satisfaction, using Likert five-point scoring method, with a total score of 50 points and a score of $<10$ is very unsatisfactory, 10-20 is unsatisfactory, 21-30 is fair, 31-40 is satisfied, and $>40$ is very satisfied. Before and after the implementation of the manoeuvrable blood collection nurse post, 200 patients were randomly selected for investigation on December 2-6, 2018 and August 7-11, 2019. The Wilcoxon rank sum test showed a significant difference in patient satisfaction ( $\mathrm{P}$ $<0.01$ ), Table 2 for details.

Table 2. Comparison of patient satisfaction before and after implementation of manoeuvrable blood collection nurse post management (n, \%).

\begin{tabular}{lllllll}
\hline Time & $\mathbf{n}$ & $\mathbf{5}$ & $\mathbf{4}$ & $\mathbf{3}$ & $\mathbf{2}$ & $\mathbf{1}$ \\
\hline Before & 200 & 38 & 128 & 26 & 6 & 2 \\
After & 200 & 46 & 144 & 10 & 0 & 0 \\
Z & & & 4.753 & 95 & \\
P & & & $<0.01$ & & \\
\hline
\end{tabular}

5, Very satisfied; 4, Satisfied; 3, Nomal; 2, Dissatisfied; 1, Very dissatisfied.

\subsection{Evaluation of Nurses' Position Management of Manoeuvrable Blood Collection Nurses}

Most of the manoeuvrable blood collection nurses are young nurses, have sufficient time and energy, and the salary is relatively low. Interviewed 20 manoeuvrable blood collection nurses by WeChat, They all expressed their willingness to take part-time manoeuvrable blood collection work. They believed that part-time work could not only increase income and ease economic pressure, but also meet 
new colleagues and understand the situation in other departments.

\section{Discussion}

The implementation of the manoeuvrable blood collection nurse post shortens the waiting time for patients to collect blood and improves patient satisfaction service quality. By implementing the post management of manoeuvrable blood collection nurses, patients waiting for blood collection during the peak blood collection period were diverted. The average waiting time for patients was shortened from 25.14 minutes to 9.41 minutes. The noisy and congested environment has been improved, nurses have reduced work pressure, and the quality of care and patient satisfaction have improved. The survey results show that after the implementation of manoeuvrable blood collection posts, patients' satisfaction with the evaluation of blood collection room services increased to $95 \%$, which was significantly higher than before $(\mathrm{P}<0.01)$.

The implementation of the manoeuvrable blood collection nurse post will revitalize the hospital's human resources and reduce the hospital's human costs. The hospital's human costs will be reflected in the employees' salaries and various benefits and subsidies [8]. Manoeuvrable blood collection nurses, as in-house nurses hired by the blood collection room, are not included in the blood collection room establishment, and the hospital does not need to increase the number of staff. The blood collection room only needs to pay for overtime, which saves basic salary, performance salary, social insurance costs, housing subsidies and other labor costs. The blood collection room of our hospital has reduced the number of 1.37 permanent nurses by setting up post management for manoeuvrable blood collection nurses, which has reduced the labor cost of the hospital.

The implementation of manoeuvrable blood collection nurse positions increases the additional income of nurses and increases the enthusiasm of the nurses. The implementation of manoeuvrable blood collection nurse positions provides young nurses with the opportunity to increase their additional income on a part-time basis, while also enabling them to gain an intuitive understanding of other departments and work experience. The implementation of manoeuvrable blood collection nurse positions has changed the traditional nursing and management model of blood collection rooms. Nurses at manoeuvrable blood collection positions can choose shifts by themselves and arrange on-the-job time based on their breaks and energy. This has promoted the enthusiasm of nurses to a certain extent [9]. In addition, the Nursing Department can investigate the working ability and attitude of young nurses by manoeuvrable job management, which can help finding nursing talents and achieve a win-win situation for individuals and hospitals [10].

At present, due to the insufficient staffing of nurses in the blood collection room of our hospital, and the opening of the second child in China, the number of maternity leave has increased, and the nurses' workload has been overloaded, leading to physical and mental fatigue, affecting the quality of care and service levels. The setting of a manoeuvrable blood collection nurse post in the outpatient blood collection room can alleviate the shortage of nursing human resources in the hospital and plays an important role in ensuring the normal operation of the nursing work [11]. At the same time, the nursing human resources in the hospital are unchanged under the condition, the manoeuvrable deployment of nursing posts can maximize the use efficiency of the existing nursing human resources, which is worthy of clinical promotion and use.

\section{Acknowledgements}

The authors declare no conflict of interest.

\section{References}

[1] Luo Yunmei, L. C., Li Haiyan, et al., Exploration and practice of mobile nurse positions in outpatient blood collection rooms. China Journal of Modern Medicine, 2014. 24 (28): p. 90-93.

[2] Haiqing, Y., The correct collection method and common errors of blood samples for clinical examination. Chinese Journal of Practical Nursing, (20): p. 34-35.

[3] Qiongxian, Z., Discussion on the Role of Blood Collection Nurses in Blood Stations. Journal of Dali University, 2012. 11 (6): p. 78-80.

[4] Wang Hong, L. Y., Discussion on Language Communication Skills between Blood Collection Nurses and Blood Donors. Chinese Community Physician (Medical Specialty), (36): p. 203.

[5] Ting, L., Discussion on Pre-post Training of New Blood Collection Nurses in Blood Station. Medical Information, 2015 (20).

[6] Meiqin, Y., The importance of quality training of blood collection nurses. Journal of Practical Hospital Clinic, 2013. 10 (3): p. 135-136.

[7] Sun Yan, X. M., Zheng Yining, Investigation and thinking on the current situation of outpatient blood draw. Chinese Nursing Management, 2013. 13 (1): p. 95-97.

[8] Yan, W., Thinking on Human Cost Management and Control in Public Hospitals. Health Economics Research, 2014 (6): p. 49-51.

[9] Hu Xuehui, Y. P., Wang Zheping, et al., Improvement and effect analysis of nursing post management mode in outpatient blood collection center. China Health Quality Management, (1).

[10] Chen Jiao, X. X., Wang Xiaoyan, et al., Study on the effect of combined management of fixed and dynamic posts in outpatient blood drawing room nursing. Journal of Nursing, (15).

[11] Wei, Z., Establishment and management of mobile nurses bank in secondary hospital. Chinese Clinical Nursing, 2014. 6 (2): p. 176-177. 